\title{
LiquidDiffract: Software for liquid total scattering analysis
}

\author{
B. J. HeINEN*, J. W. E. DREWITT, O. T. LORD
}

School of Earth Sciences, University of Bristol, BS8 1RJ, UK

(*correspondence: benedict.heinen@bristol.ac.uk)

Despite a lack of long-range order, liquids and glasses possess a variety of short to medium range order, originating from chemical interactions that lead to local structure. Fluids and melts can occur from the crust to the core and are key to our understanding of the dynamic Earth, including mass transfer in the deep interior, and geothermal, volcanic, and ore-forming processes at the near surface. The density and viscosity of transient melts in the mantle controls gravity driven melt transport, regulating large scale volatile fluxes and metasomatism.

Diffraction provides a direct measure of atomic-scale structure. Diffuse scattering, that is normally subtracted away as 'background' during Rietveld refinement, contains information on static disorder and average local structure. The intensity of total scattering from a sample is due to both coherent and incoherent (Compton) scattering of radiation. The Compton scattering from a sample can be predicted analytically, leaving a direct measure of coherent scattering intensity, which is related to the static structure factor, $S(Q)$, by a normalisation factor. The Fourier transform of a normalised $S(Q)$ gives the pair distribution function, $g(r)$, which describes the probability of finding an atom at a distance $(r)$ from another atom. The areas of the peaks in $g(r)$ are proportional to the coordination numbers of atomic pairs, and the slope of $g(r)$ below the first interatomic distance $\left(r_{0}\right)$ gives the number density, $\rho$.

In this contribution we describe LiquidDiffract [1], an open source, Python based API and GUI that provides an easy to use interface to process total diffraction data of amorphous materials. LiquidDiffract implements the iterative procedure proposed by Eggert et al. [2], which minimises $S(Q)$ normalisation errors by removing non-physical oscillations in $g(r<r)$. This provides a normalised $S(Q)$ and $g(r)$, whilst simultaneously refining the number density. LiquidDiffract works with both X-ray and neutron diffraction data. The efficacy of LiquidDiffract has been demonstrated on measurements of structural ordering in liquid gallium at extreme conditions [3]. Our software can be used for monatomic (e.g. liquid metals) and polyatomic (e.g. silicate melts) samples of any amorphous material.

[1] Heinen (2019) doi:10.5281/zenodo.3592511 [2] Eggert et al. (2002) Phys. Rev. B 65, 174105. [3] Drewitt et al. (2020), submitted. 